\title{
An Exact Solution for Instantaneous Axisymmetric Flow of Polymers
}

\author{
Sergei ALEXANDROV, Milan ZUTKOVIC, Van Manh DINH
}

\begin{abstract}
The main objective of the present paper is to study, by means of a problem permitting a semi-analytic solution, the qualitative behaviour of solution for a model that is used for describing deformation of incompressible polymers near frictional interfaces. It is assumed that the regime of sticking occurs at the friction interfaces. The constitutive equations of the model are a pressure - dependent yield criterion and a flow rule. Such features of the solutions as non-existence and singularity are emphasized.
\end{abstract}

Keywords: friction; plasticity; rough wall; singularity

\section{INTRODUCTION}

Experimental studies demonstrate that skin layers are often generated in the vicinity of frictional interfaces in injecting molding of polymers. A review of such experimental results is provided in [1]. It is known from the theory of plasticity for metals that the equivalent strain rate approaches infinity near maximum friction surfaces [2-4]. This feature of solution behaviour is used to describe the generation of fine grain layers in metal forming processes [5]. It is of interest to extend this approach to processing of polymers. To this end, it is necessary to understand whether or not the behaviour of solutions for models that are used for polymers is similar to that found in [2-4]. In the present paper, the yield criterion for polyethylene proposed in [6] is adopted. This yield criterion is pressure-dependent. However, the volume change of polyethylene is similar to that observed in metals [6]. Therefore, it is reasonable to assume that the equation of incompressibility is satisfied.

In order to study the behaviour of solutions near frictional interfaces, it is not necessary to solve a boundary value problem of practical significance. It is more important to study an exact solution (i.e. the solution that exactly satisfies all the equations and boundary conditions). An ideal plane strain boundary value problem consists of a deformation comprising the simultaneous shearing and expansion (or contraction) of a hollow cylinder [7-9]. An axisymmetric analogue to this problem is a deformation comprising the simultaneous shearing and expansion (or contraction) of a material between two conical surfaces. This boundary value problem is solved in the present paper. The solution is for instantaneous flow. Most of the analytic and semi-analytic rigid plastic solutions other than solutions for stationary problems belong to this type of flow [10-12]. A rare case of the rigid plastic solutions for both instantaneous and continued flows is presented in [13].

\section{CONSTITUTIVE EQUATIONS}

The linear and quadratic stress invariants, $I_{1}$ and $I_{2}$, may be defined as

$$
I_{1}=\sigma_{1}+\sigma_{2}+\sigma_{3}, \quad I_{2}=\sqrt{\frac{3}{2}\left(\tau_{1}^{2}+\tau_{2}^{2}+\tau_{3}^{2}\right)}
$$

where $\sigma_{1}, \sigma_{2}$ and $\sigma_{3}$ are the principal stresses. Moreover, $\tau_{1}=\sigma_{1}-I_{1} / 3, \quad \tau_{2}=\sigma_{2}-I_{1} / 3$ and $\tau_{3}=\sigma_{3}-I_{1} / 3$. The yield criterion for polyethylene proposed in [6] is

$I_{2}+c_{1} I_{1}=c$

where $c$ and $c_{1}$ are material constants. This material is practically incompressible [6]. Therefore, the plastic potential function of Mises is adopted in the present paper. In this case the flow rule results in

$\xi_{1}=\lambda \tau_{1}, \quad \xi_{2}=\lambda \tau_{2}, \quad \xi_{3}=\lambda \tau_{3}$

where $\xi_{1}, \xi_{2}$ and $\xi_{3}$ are the principal strain rates and $\lambda$ is a non-negative multiplier. In addition to (3), the constitutive equations require that the stress and strain tensors are coaxial. It follows from Eq. (3) that

$\xi_{1}+\xi_{2}+\xi_{3}=0$

\section{STATEMENT OF THE PROBLEM}

Consider a conical layer of plastic material between two conical surfaces of total angles $2 \theta_{1}$ and $2 \theta_{2}$. It is convenient to introduce a spherical coordinate system $(r, \theta, \phi)$ with the axis $\theta=0$ coinciding with the axis of symmetry of the conical surfaces (Fig. 1). The plastic material occupies the domain $0 \leq r<\infty, \theta_{1} \leq \theta \leq \theta_{2}$ and $0 \leq \phi \leq 2 \pi$. Let $u_{r}, u_{\theta}$ and $u_{\phi}$ be the velocity components referred to this coordinate system. The rate of increasing of the angle $\theta_{1}$ will be denoted by $\omega_{1}$. Then,

$u_{\theta}=\omega_{1} r$

for $\theta=\theta_{1}$. The conical surfaces are friction surfaces. It is assumed that the regime of sticking occurs at these surfaces. The internal conical surface is fixed against rotation. Therefore,

$u_{\phi}=0$ 
for $\theta=\theta_{1}$. The external conical surface rotates with an angular velocity $\omega_{2}$.

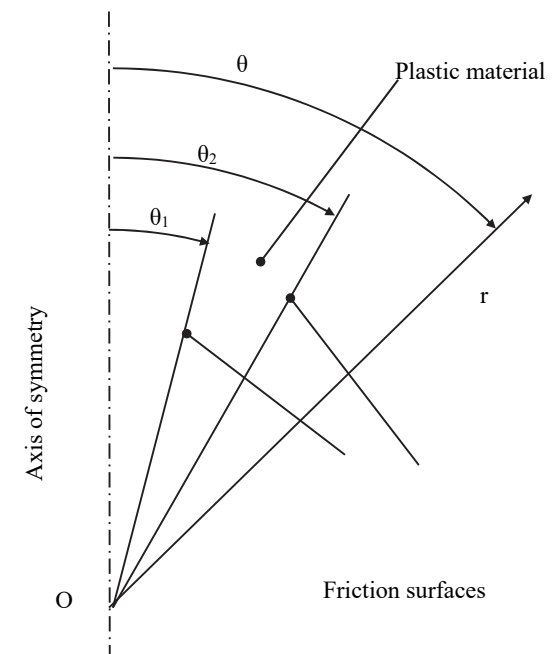

Figure 1 Deformation of plastic material between conical surfaces

Therefore,

$u_{\phi}=r \omega_{2} \sin \theta_{2}$

for $\theta=\theta_{2}$. Let $\xi_{r r}, \xi_{\theta \theta}, \xi_{\phi \phi}, \xi_{r \theta}, \xi_{r \phi}$ and $\xi_{\theta \phi}$ be the components of the strain rate tensor referred to the spherical coordinate system. It is possible to assume with no loss of generality that $\omega_{2}>0$. Then,

$\xi_{\theta \phi}>0$.

Let $\sigma_{r r}, \sigma_{\theta \theta}, \sigma_{\phi \phi}, \sigma_{r \theta}, \sigma_{r \phi}$ and $\sigma_{\theta \phi}$ be the components of the stress tensor referred to the spherical coordinate system. The boundary conditions formulated show that the solution is independent of $\phi$. Then, the equilibrium equations are

$\frac{\partial \sigma_{r r}}{\partial r}+\frac{\partial \sigma_{r \theta}}{r \partial \theta}+\frac{2 \sigma_{r r}-\sigma_{\theta \theta}-\sigma_{\phi \phi}+\sigma_{r \theta} \cot \theta}{r}=0$,

$\frac{\partial \sigma_{r \theta}}{\partial r}+\frac{\partial \sigma_{\theta \theta}}{r \partial \theta}+\frac{\left(\sigma_{\theta \theta}-\sigma_{\phi \phi}\right) \cot \theta+3 \sigma_{r \theta}}{r}=0$,

$\frac{\partial \sigma_{r \phi}}{\partial r}+\frac{\partial \sigma_{\theta \phi}}{r \partial \theta}+\frac{2 \sigma_{\theta \phi} \cot \theta+3 \sigma_{r \phi}}{r}=0$.

The strain rate components are expressed in terms of the velocity components as

$$
\begin{aligned}
& \xi_{r r}=\frac{\partial u_{r}}{\partial r}, \quad \xi_{\theta \theta}=\frac{1}{r}\left(\frac{\partial u_{\theta}}{\partial \theta}+u_{r}\right), \\
& \xi_{\phi \phi}=\frac{1}{r \sin \theta}\left(u_{r} \sin \theta+u_{\theta} \cos \theta\right), \\
& \xi_{\theta \phi}=\frac{1}{2 r \sin \theta}\left(\sin \theta \frac{\partial u_{\phi}}{\partial \theta}-u_{\phi} \cos \theta\right), \\
& \xi_{r \phi}=\frac{1}{2}\left(\frac{\partial u_{\phi}}{\partial r}-\frac{u_{\phi}}{r}\right), \quad \xi_{r \theta}=\frac{1}{2}\left(\frac{\partial u_{\theta}}{\partial r}-\frac{u_{\theta}}{r}+\frac{\partial u_{r}}{r \partial \theta}\right) .
\end{aligned}
$$

\section{GENERAL SOLUTION}

It is reasonable to seek the general velocity of the form

$u_{r}=0, \quad u_{\theta}=r W_{\theta}(\theta), \quad u_{\phi}=r W_{\phi}(\theta)$

where $W_{\theta}(\theta)$ and $W_{\phi}(\theta)$ are arbitrary functions of $\theta$. Substituting Eq. (11) into (10) yields

$$
\begin{aligned}
& \xi_{r r}=0, \quad \xi_{\theta \theta}=\frac{d W_{\theta}}{d \theta}, \quad \xi_{\phi \phi}=W_{\theta} \cot \theta, \\
& \xi_{\theta \phi}=\frac{\sin \theta}{2} \frac{d}{d \theta}\left(\frac{W_{\phi}}{\sin \theta}\right), \quad \xi_{r \phi}=0, \quad \xi_{r \theta}=0 .
\end{aligned}
$$

Therefore, one of the principal strain rate (and stress) directions coincides with the $r$ - direction. It is possible to assume with no loss of generality that $\sigma_{r r} \equiv \sigma_{3}$ and $\xi_{r r} \equiv \xi_{3}$. Then, the principal stresses $\sigma_{1}$ and $\sigma_{2}$ are in the $(\theta, \phi)$ plane. Let $\varphi$ be the inclination of the principal stress direction corresponding to the stress $\sigma_{1}$ to the $\theta$-direction. It follows from the transformation equations for stress components that

$$
\begin{aligned}
\tau_{\theta \theta} & =-\frac{\tau_{3}}{2}+\frac{\left(\tau_{1}-\tau_{2}\right) \cos 2 \varphi}{2}, \\
\tau_{\phi \phi} & =-\frac{\tau_{3}}{2}-\frac{\left(\tau_{1}-\tau_{2}\right) \cos 2 \varphi}{2}, \\
\sigma_{\theta \phi} & =\frac{\left(\tau_{1}-\tau_{2}\right) \sin 2 \varphi}{2} .
\end{aligned}
$$

It has been taken into account here that $\tau_{1}+\tau_{2}+\tau_{3} \equiv 0$. Also, $\tau_{\theta \theta}=\sigma_{\theta \theta}-\sigma$ and $\tau_{\phi \phi}=\sigma_{\phi \phi}-\sigma$ where $\sigma$ is the hydrostatic stress. It is seen from Eq. (1) that $I_{1}=3 \sigma$. It follows from Eqs. (3) and (12) that $\tau_{3}=0$. Then, $\tau_{1}+\tau_{2}=0$ and Eq. (13) becomes

$\tau_{\theta \theta}=\tau_{1} \cos 2 \varphi, \quad \tau_{\phi \phi}=-\tau_{1} \cos 2 \varphi, \quad \sigma_{\theta \phi}=\tau_{1} \sin 2 \varphi$.

Since the model is coaxial, it is evident from Eq. (12) that $\sigma_{r \phi}=\sigma_{r \theta}=0$. Substituting these equations and Eq. (14) into Eq. (9) yields 
$\frac{\partial \sigma}{\partial r}=0, \quad \frac{\partial \sigma}{\partial \theta}+\frac{\partial\left(\tau_{1} \cos 2 \varphi\right)}{\partial \theta}+2 \tau_{1} \cos 2 \varphi \cot \theta=0$

$\frac{\partial \sigma_{\theta \phi}}{\partial \theta}+2 \sigma_{\theta \phi} \cot \theta=0$

The third equation can be immediately integrated to give

$$
\sigma_{\theta \phi}=\frac{c p}{\sin ^{2} \theta}
$$

where $p$ is a constant of integration. It is seen from Eq. (8) that $\sigma_{\theta \phi}>0$ and, therefore, $p>0$. The first equation in Eq. (15) shows that $\sigma$ is independent of $r$. Then, the second equation becomes

$\frac{\mathrm{d} \sigma}{\mathrm{d} \theta}+\frac{\mathrm{d}\left(\tau_{1} \cos 2 \varphi\right)}{\mathrm{d} \theta}+2 \tau_{1} \cos 2 \varphi \cot \theta=0$

It has been assumed that both $\tau_{1}$ and $\varphi$ are independent of $r$. It is possible to assume with no loss of generality that $\tau_{1}>0$. This inequality together with the inequality $\sigma_{\theta \phi}>0$ and Eq. (14) shows that

$0<\varphi<\pi / 2$

Since $\tau_{3}=0$, it follows from Eq. (1) that $I_{2}=\sqrt{3} \tau_{1}$. Then, Eq. (2) becomes

$\sqrt{3} \tau_{1}+3 c_{1} \sigma=c$

It follows from Eqs. (14), (16) and (19) that

$\tau_{1}=\frac{c p}{\sin 2 \varphi \sin ^{2} \theta}, \quad \sigma=\frac{c}{3 c_{1}}-\frac{c p}{\sqrt{3} c_{1} \sin 2 \varphi \sin ^{2} \theta}$

Eqs. (17) and (20) combine to give

$\frac{\mathrm{d} \varphi}{\mathrm{d} \theta}=\frac{\cot \theta \sin 2 \varphi}{\sqrt{3} c_{1}-\cos 2 \varphi}$

Integrating results in to give

$C_{2} \sin \theta=\frac{\tan ^{n} \varphi}{\sqrt{\sin 2 \varphi}}$

where $C_{2}$ is a constant of integration and $n=\sqrt{3} c_{1} / 2$. The incompressibility Eq. (4) can be rewritten as $\xi_{r r}+\xi_{\theta \theta}+\xi_{\phi \phi}=0$. Using Eq. (12) transforms this equation to $\mathrm{d} W_{\theta} / \mathrm{d} \theta+W_{\theta} \cot \theta=0$. This equation can be immediately integrated to give

$W_{\theta}=\frac{W_{0}}{\sin \theta}$ where $W_{0}$ is a constant of integration. It follows from Eqs. (5), (11) and (23) that $W_{0}=\omega_{1} \sin \theta_{1}$. Then, Eq. (23) becomes

$W_{\theta}=\frac{\omega_{1} \sin \theta_{1}}{\sin \theta}$

A consequence of Eq. (3) is $\xi_{\theta \theta}=\lambda \tau_{\theta \theta}$ and $\xi_{\theta \phi}=\lambda \sigma_{\theta \phi}$. Eliminating $\lambda$ in these equations yields $\xi_{\theta \theta} \sigma_{\theta \phi}=\xi_{\theta \phi} \tau_{\theta \theta}$. Using Eqs. (12), (14) and (24) leads to

$\frac{\mathrm{d}}{\mathrm{d} \theta}\left(\frac{W_{\phi}}{\sin \theta}\right)=-\frac{2 \omega_{1} \sin \theta_{1} \tan 2 \varphi \cos \theta}{\sin ^{3} \theta}$.

It is seen from Eqs. (6) and (11) that $W_{\phi}=0$ at $\theta=\theta_{1}$. The solution of Eq. (25) satisfying this boundary condition can be written as leads to

$W_{\phi}=-2 \omega_{1} \sin \theta_{1} \sin \theta \int_{\theta_{1}}^{\theta} \frac{\tan 2 \varphi \cos \beta}{\sin ^{3} \beta} \mathrm{d} \beta$.

It is understood here that $\varphi$ is a function $\beta$ found from Eq. (22). It follows from Eq. (7) and (11) that $W_{\phi}=\omega_{2} \sin \theta_{2}$ at $\theta=\theta_{2}$. This boundary condition and Eq. (26) combine to give

$\omega_{2}=-2 \omega_{1} \sin \theta_{1} \int_{\theta_{1}}^{\theta_{2}} \frac{\tan 2 \varphi \cos \theta}{\sin ^{3} \theta} \mathrm{d} \theta$.

\section{ANALYSIS OF THE SOLUTION}

The denominator on the right hand side of Eq. (21) vanishes at

$\varphi=\varphi_{c}=\frac{1}{2} \arccos \left(\sqrt{3} c_{1}\right)$

It is seen from Eq. (26) that $\omega_{1}>0$ if $\tan 2 \varphi<0$ in the range $\theta_{1} \leq \theta \leq \theta_{2}$ and $\omega_{1}<0$ if $\tan 2 \varphi>0$ in the range $\theta_{1} \leq \theta \leq \theta_{2}$. These two cases should be treated separately. Firstly, assume that $\omega_{1}>0$. Then, taking into account Eq. (18) it is possible to find that the value of $\varphi$ varies in the range $\pi / 4 \leq \varphi<\pi / 2$. It is evident that $\varphi_{c}$ does not belong to this range. Therefore, the denominator on the right hand side of Eq. (21) never vanishes, $\mathrm{d} \varphi / \mathrm{d} \theta>0$ in the range $\theta_{1} \leq \theta \leq \theta_{2}$ and $\varphi$ may attain the value of $\pi / 4$ at $\theta=\theta_{1}$. In this case, it follows from Eq. (27) that $\omega_{2} \rightarrow \infty$ as $\theta \rightarrow \theta_{1}$. Therefore, the solution at sticking always exists independently of other conditions.

Assume that $\omega_{1}<0$. Then, taking into account Eq. (18) it is possible to find that the value of $\varphi$ varies in the range $0 \leq \varphi<\pi / 4$. The value of $\varphi_{c}$ belongs to this range. Moreover, it is seen from Eq. (22) that $\theta$ considered 
as a function of $\varphi$ attains a minimum at $\varphi=\varphi_{c}$. Therefore, $\varphi=\varphi_{c}$ at $\theta=\theta_{1}$. Using these conditions it is possible to find $C_{2}$ involved in Eq. (22) and this equation becomes

$\sin \theta=\frac{\tan ^{n} \varphi}{\sqrt{\sin 2 \varphi}} \frac{\sqrt{\sin 2 \varphi_{c}} \sin \theta_{1}}{\tan ^{n} \varphi_{c}}$.

Using this equation the integral in Eq. (27) can be evaluated giving a maximum possible value of $\omega_{2}$. No solution at sticking exists at lager values of $\omega_{2}$. Consider the behavior of the strain rate component $\xi_{\theta \phi}$ when $\varphi=\varphi_{c}$ at $\theta=\theta_{1}$. It follows from Eqs. (12) and (21) that

$\xi_{\theta \phi}=\frac{\sin \theta}{2} \frac{\mathrm{d}}{\mathrm{d} \theta}\left(\frac{W_{\phi}}{\sin \theta}\right)=\frac{\sin \theta \cot \theta \sin 2 \varphi}{2\left(\sqrt{3} c_{1}-\cos 2 \varphi\right)} \frac{\mathrm{d}}{\mathrm{d} \varphi}\left(\frac{W_{\phi}}{\sin \theta}\right)$

It is seen from this equation and Eq. (28) that $\left|\xi_{\theta \phi}\right| \rightarrow \infty$ as $\theta \rightarrow \theta_{1}$. This feature of the solution predicts the existence of a layer of intensive deformation in the vicinity of this friction surface. This deformation may be responsible for the generation of skin layers [1].

\section{CONCLUSIONS}

Using the boundary value problem permitting a semianalytic solution the qualitative behavior of the solution in the vicinity of the friction interface for the yield criterion proposed in [6] has been studied. The main contribution of the present paper is the exact asymptotic analysis of solution behavior in the vicinity of the friction surface. Using this asymptotic analysis, the following conclusions can be drawn.

1. In the case of $\omega_{1}>0$ (Fig. 1), the solution satisfying the regime of sticking at the inner conical surface exists independently of the other boundary conditions.

2. In the case of $\omega_{1}<0$ (Fig. 1), the solution satisfying the regime of sticking does not exist if the value of $\omega_{2}$ is large enough.

3. In the case of $\omega_{1}<0$ (Fig. 1), the solution is singular, $\left|\xi_{\theta \phi}\right| \rightarrow \infty$ as $\theta \rightarrow \theta_{1}$, at the transition from the regime of sticking to the regime of sliding at the surface $\theta=\theta_{1}$.

4. The singularity in the velocity field may describe the generation of skin layers.

\section{Acknowledgements}

The authors acknowledge financial support of this research through grants RFBR-17-51-540003 (Russia) and VAST.HTQT.NGA.07/17-18 (Vietnam).

\section{REFERENCES}

[1] Pantani, R., Coccorullo, I., Speranza, V., \& Titomanlio, G. (2005). Modeling of morphology evolution in the injection molding process of thermoplastic polymers. Progress in Polymer Science, 30(12), 1185-1222. https://doi.org/10.1016/j.progpolymsci.2005.09.001

[2] Alexandrov, S. \& Richmond, O. (2001). Singular plastic flow fields near surfaces of maximum friction stress. International Journal of Non-Linear Mechanics, 36(1), 1-11. https://doi.org/10.1016/S0020-7462(99)00075-X

[3] Alexandrov, S. \& Jeng, Y. R. (2013). Singular rigid/plastic solutions in anisotropic plasticity under plane strain conditions. Continuum Mechanics and Thermodynamics, 25(5), 685-689. https://doi.org/10.1007/s00161-013-0304-y

[4] Alexandrov, S. \& Mustafa, Y. (2013). Singular solutions in viscoplasticity under plane strain conditions. Meccanica, 48(9), 2203-2208. https://doi.org/10.1007/s11012-013-9736-5

[5] Goldstein, R. V. \& Alexandrov, S. E. (2015). An approach to prediction of microstructure formation near friction surfaces at large plastic strains. Physical Mesomechanics, 18(5), 223-227. https://doi.org/10.1134/S1029959915030066

[6] Spitzig, W. A. \& Richmond, O. (1979). Effect of hydrostatic pressure on the deformation behavior of polyethylene and polycarbonate in tension and in compression. Polymer Engineering \& Science, 19(6), 1129-1139. https://doi.org/10.1002/pen.760191602

[7] Alexandrov, S. \& Harris, D. (2006). Comparison of solution behaviour for three models of pressure-dependent plasticity: A simple analytical example. International Journal of Mechanical Sciences, 48(7), 750-762. https://doi.org/10.1016/j.jmecsci.2006.01.009

[8] Alexandrov, S. \& Mishuris, G. (2009). Qualitative behaviour of viscoplastic solutions in the vicinity of maximum-friction surfaces. Journal of Engineering Mathematics, 65(2), 143156. https://doi.org/10.1007/s10665-009-9277-z

[9] Lyamina, E. A. \& Date, P. P. (2016). Planar plastic flow of polymers near very rough walls. Structural Engineering and Mechanics, 58(4), 707-718. https://doi.org/10.12989/sem.2016.58.4.707

[10] Hill, R. (1950). The mathematical theory of plasticity. Oxford: Clarendon Press.

[11] Druyanov, B. (1993).Technological mechanics of porous bodies. New-York: Clarendon Press.

[12] Rees, D. W. A. (2006). Basic Engineering Plasticity. Amsterdam: Elsevier. https://doi.org/10.1016/B978-0-7506-8025-7.50009-X

[13] Collins, I. F. \& Meguid, S. A. (1977). On the influence of hardening and anisotropy on the plane-strain compression of thin metal strip. Journal of Applied Mechanics Transactions ASME, 44(2), 271-278. https://doi.org/10.1115/1.3424037

\section{Contact information:}

Sergei ALEXANDROV, PhD, Professor

Ishlinsky Institute for Problems in Mechanics, Russian Academy of Sciences 101-1 Prospect Vernadskogo, 119526 Moscow, Russia

E-mail: sergei_alexandrov@yahoo.com

Federal State Autonomous Educational Institution of Higher Education

"South Ural State University (national research university)"

76, Lenin prospekt, Chelyabinsk, Russia, 454080

E-mail: alexandrovse@susu.ru

Milan ZUTKOVIC, MSc, Phd student

(Corresponding author)

Faculty of Technical Sciences, University of Novi Sad,

Trg Dositeja Obradovica 6, 21000 Novi Sad, Serbia

E-mail: zutkovic@gmail.com

Van Manh DINH, PhD, Professor

Institute of Mechanics, Vietnam Academy of Science and Technology, 264 Doi Can, Ba dinh, Hanoi, Vietnam

E-mail: dvmanh@imech.ac.vn 